Interleukin- 6 assays can be useful in diagnosis of sepsis alongside CRP. This study looks at the influence of IL- 6 and CRP results on clinical decision making.

Methods A prospective web-based questionnaire survey of both junior doctors (online survey) and Consultants (focus group) was carried out using 20 hypothetical scenarios of neonatal sepsis along with hypothetical IL- 6 and CRP results. The differences in diagnostic certainty of sepsis on the basis of clinical history alone were compared with that of addition of CRP and IL- 6 results, within and between both the trainee and expert groups. (Expert group consensus responses were considered as gold-standard).

Results Experts: Based on clinical history, CRP and IL- 6 results, experts agreed to the possibility of sepsis in only $25 \%$ of the clinical situations. Antibiotic usage by experts subsequent to sepsis categorisation was reduced with the availability of CRP results. $(55 \%$ after IL-6 vs. 30\% after CRP results).

Trainees: CRP results were shown to be statistically significant in changing clinician's decisions. Trainees favoured a greater likelihood of sepsis when IL-6 results were available prior to CRP results. Using the focus group consensus as gold standard, IL- 6 results were used by trainees for confirming sepsis irrespective of whether they were available prior to or after CRP results.

Conclusion Both point-of-care IL- 6 test results and CRP results helped doctors in confirming a diagnosis of sepsis. IL-6 was not useful in ruling out sepsis.

\section{HIGH INTESTINAL MUCOSAL INJURY ASSOCIATING WITH LOW ANTI ENDOTOXINE IMIMUNITY IN VERY LOW BIRTH WEIGHT INFANTS}

doi:10.1136/archdischild-2012-302724.1187

N Panakhova, S Hasanov, S Alasgarova, K Shafiyeva. Azerbaijan Medical University, Baku, Azerbaijan

Background and Aims Premature infants are exposed to numerous perinatal stresses such as hypothermia, hypoxia, hypotension, umbilical vessel catheterization. All of these have been postulated as risk factors for ischemic injury of the neonatal intestine. The intestinal permeability is increased in bacterial translocation which can lead to endotoxemia and multiple organ failure. The aim of this study was to determine anti endotoxine immunity (AEI) in premature infant depend on birth weight.

Methods Premature newborns were divided into two groups. The first group consisted of 61 newborns with birth weight more than 1500 gram and 20 infant with birth weight less than 1500 gram were included in second study. In this study urinary intestinal fatty acid bind protein (i-FABP) level was measured as a specific marker for intestinal mucosal damage and serum LBP concentration was detected for estimation of AEI. Both markers were determined by enzyme linked immunsorbent assay.

Results The mean i-FABP concentration in the second group $(1.75 \pm 0.62 \mathrm{ng} / \mathrm{ml})$ was elevated in 1.4 times compared with the first group $(1.23 \pm 0.23 \mathrm{ng} / \mathrm{ml})$. Significant high urine i-FABP concentration was observed in died infants of second group $(2.39 \pm 0.88 \mathrm{ng} / \mathrm{ml}$, $\mathrm{p}<0.05)$. In contrast the serum LBP level in newborns of second group was lower $(23.1 \pm 4.5 \mathrm{ng} / \mathrm{ml})$ in 1.4 time compared to newborns of first group $(32.1 \pm 2.3 \mathrm{ng} / \mathrm{ml})$.

Conclusion Very low birth weight newborns are at increased risk of intestinal mucosal injury and endotoxemia and decreased serum LBP level in these infants should be considered as an unfavorable factor for sepsis.

\section{SERIAL QUANTITATIVE C-REACTIVE PROTEIN (QCRP) CONCENTRATIONS TO DETERMINE APPROPRIATENESS OF ANTIBIOTICS IN NEONATAL SEPSIS: A NESTED CASE CONTROL STUDY}

ISS Patil, IP Kumar, ${ }^{2} \mathrm{P}$ Ray, ${ }^{3} \mathrm{~S}$ Verma. 'Pediatrics; ${ }^{2}$ Microbiology; ${ }^{3}$ PediatricsBiochemistry, Post Graduate Institute of Medical Education and Reasearch (PGIMER), Chandigarh, India

Background and Aims QCRP being acute phase reactant has predictable pattern of rise and fall following inflammation. Few studies have used OCRP for appropriateness of antibiotic therapy.

To determine the difference in the magnitude of change in QCRP values from baseline to $48 \mathrm{~h}$ in subjects with culture positive neonatal sepsis receiving sensitive antibiotics (CPSA) versus those receiving resistant antibiotics (CPRA).

Methods Neonates < twenty-eight days with suspected sepsis and baseline QCRP $>10 \mathrm{mg} / \mathrm{L}$ were enrolled. Serum samples at 24, 36 and $48 \mathrm{~h}$ after initiation of antibiotics were analyzed for QCRP (PETIA: Particle enhanced turbidimetric immunoassay). After collecting blood culture [BD BACTEC TM Peds Plus/F] report, CPSA and CPRA were cases and sterile cultures were controls. Mann-Whitney U test, linear regression, ROC curve and Youden's index were used to measure appropriateness of antibiotic therapy.

Results In one hundred forty-one sepsis episodes forty-five were CPSA, forty-four were CPRA and fifty two were culture sterile. The difference in QCRP between CPSA and CPRA was significant at all time points $(p<0.001)$. The area under ROC curve was highest for $\Delta \mathrm{CRP}_{0-48}[\mathrm{CRP}(0 \mathrm{hr})-\mathrm{CRP}(48 \mathrm{hr})]$ and $\Delta \mathrm{CRP}_{24-48}[\mathrm{CRP}(24 \mathrm{hr})$-CRP $(48 \mathrm{hr}$ )] i.e 0.879 (CI: $0.80,0.95)$ and 0.89 (CI: 0.81, 0.96) respectively. If $\Delta \mathrm{CRP}_{0-48}$ was $\geq 6.2 \mathrm{mg} / \mathrm{L}$, the infant was likely to be getting sensitive antibiotics (sensitivity $86 \%$, specificity $84 \%$ ).

Conclusion A decrease in serum QCRP by $6.2 \mathrm{mg} / \mathrm{L}$ can be used as a useful indicator of the appropriateness of antibiotics in neonatal sepsis.

\section{MISSED OPPORTUNITIES FOR PREVENTION OF EARLY ONSET GROUP B STREPTOCOCCUS (EOGBS) INFECTION IN NORTHERN IRELAND}

doi:10.1136/archdischild-2012-302724.1189

'S Magowan, ${ }^{1} \mathrm{~J}$ Lewis, ${ }^{2} \mathrm{~S}$ Craig, ${ }^{3} \mathrm{G}$ Ong, ${ }^{4} \mathrm{R}$ Ashe. ${ }^{1}$ Royal Belfast Hospital for Sick Children; ${ }^{2}$ Neonatology, Royal Maternity Hospital; ${ }^{3}$ Microbiology, Royal Victoria Hospital, Belfast; ${ }^{4}$ Obstetrics, Antrim Area Hospital, Antrim, UK

Background and Aims EOGBS infection is associated with high morbidity and mortality. Historically N. Ireland has had a higher incidence than other parts of the UK. The incidence can be reduced by intrapartum antibiotic prophylaxis (IAP). The RCOG (UK) 2003 guideline on prevention of EOGBS disease states that IAP should be considered in the presence of $\geq 2$ risk factors for GBS. We sought to determine (1) the 2008-2010 incidence of EOGBS disease in N Ireland and (2) whether opportunities to give IAP were missed?

Methods Infants with positive blood or CSF cultures for EOGBS during 2008-2010, were identified by laboratory staff. Data was retrieved from maternal and neonatal charts of affected babies.

Results 35 infants had EOGBS infection. This gave an incidence of $0.47 / 1000$ live births. (England \& Wales incidence $=0.34 / 1000$ live births). Four infants died; 3 due to EOGBS. Data was missing from 3 mothers. 10/32 mothers had 1 risk factor and $4 / 10$ received IAP. $8 / 32$ mothers had $\geq 2$ risk factors; $4 / 8$ received intrapartum antibiotics but only 1 as per the RCOG (UK) guidelines. One baby from this group died.

Conclusions Our data suggests that EOGBS infection rate remains higher than other parts of the UK. There appear to be significant missed opportunities for IAP. This may pertain to uncertainties in interpreting RCOG EOGBS prevention guidelines. OXYGEN INTERMEDIATES IN PRETERM NEONATES

doi:10.1136/archdischild-2012-302724.1190 
${ }^{1,2,3} \mathrm{HO}$ Eliwan, ${ }^{3} \mathrm{~W}$ Watson, ${ }^{3} \mathrm{~A}$ O'Neill, $2,3 \mathrm{~F}$ O'Hare, $1,3,4,5 \mathrm{EJ}$ Molloy. ${ }^{1}$ Neonatology, Our Lady's Children's Hospital; ${ }^{2}$ Neonatology, National Maternity Hospital, Holles Street; ${ }^{3} U C D$ School of Medicine and Medical Science, Conway Institute for Biomolecular and Biomedical Science, University College Dublin; ${ }^{4}$ National Maternity Hospital, Holles Street; ${ }^{5}$ Royal College of Surgeon, Dublin, Ireland

Background Inflammation and infection are important aetiological factors in development of preterm birth. Inflammation is associated with many disorders of preterm infants including periventricular leukomalacia, chronic lung disease and necrotising eneterocolitis.

Aims To compare neutrophil and monocyte responses to lipopolysaccaride (LPS) +/-APC (activated protein c) stimulation in preterm neonates $<32$ weeks gestation with adults controls.

Methods Whole blood was incubated with LPS +/-APC and Tolllike receptor4 (TLR4), CD11b expression, and reactive oxygen intermediate (ROI) release from neutophils and monocytes was examined by flow cytometry.

Results Both adults $(n=15)$ and preterm neonates $(n=30)$ had significantly increased LPS induced neutrophil CD11b expression but preterms are less responsive than adults. There was a significant increase in neutrophil ROI in response to LPS in adults and preterm neonates on day 1 and this was significantly reduced by APC There was significant higher baseline and endotoxin response of monocyte ROI in preterm neonates compared to adult $(p<0.05)$. However APC had not reduced this response.

Conclusion Increased ROI release may mediate tissue damage and was significantly increased in preterm neonates and adults. APC reduced LPS-induced neutrophil ROI release. This may benefit preterm neonates at high risk of multiorgan inflammatory disorders but they are at high risk of haemorrhage. Further examination of APC mutants with anti-inflammatory but decreased anticoagulant properties is merited.

\section{LATE ONSET SEPSIS IN PREMATURE INFANTS. ARE WE ABLE TO PREDICT WHO IS SEPTIC OR NOT?}

doi:10.1136/archdischild-2012-302724.1191

K Gorman, M Dominguez, N Mc Callion. Department of Paediatrics, Rotunda Hospital/ Royal College of Surgeons in Ireland, Dublin, Ireland

Aim To prospectively assess staff prediction of culture positivity, clinical signs noted and suggested duration of antibiotic therapy required at the time of septic screen in premature infants suspected of having late onset sepsis.

Methods This was a prospective study involving anonymous staff questionnaires filled out by both nursing and medical staff at the time of septic screens performed for suspected late onset sepsis in the neonatal intensive care unit (NICU) of Rotunda Maternity Hospital, Dublin from October 2009 to 2010. Eligibility criteria was defined as premature infants ( $<34$ weeks gestation) and $>5$ days old undergoing septic work up for suspicion of infection. Prospective review of all blood, urine and CSF cultures obtained from the neonates. Staff opinion on the likelihood of positive BC was correlated with laboratory results and treatment course.

Results Total of 60 surveys collected in the twelve month period. Information is available from 56 septic work ups carried out on 37 infants during twelve month period on infants who fulfilled the criteria. Doctors correctly guessed if the infant was septic or not at the time of work up $58.3 \%$ compared to $56.3 \%$ of nursing staff. There was no stasticial significance between C-reactive protein, white cell or neutrophils counts between positive and negative cultures.

Conclusion Experienced doctors and nurses were unable to accurately predict which neonate would have a positive culture. This highlights the difficulty in the NICU setting of judging correctly who is septic in very low birth weight infants.

\section{TITLE: WBC COUNT IN PROBABLE AND CULTURE PROVEN NEONATAL SEPSIS, AND IT'S AFFECT ON MORTALITY}

doi:10.1136/archdischild-2012-302724.1192

MS Ahmad, Neonatology. Pediatrics, Fazl-e-Omar Hospital, Chenab Nagar(Rabwah), Pakistan

Aims The aim of this study was to ascertain the prevalence of high and low WBC count on admission among the cases of probable and culture proven neonatal sepsis, and its affect on mortality rate.

Methods WBC count (by Madonic CA 620 analyzer) of all patients admitted in NICU, Fazl-e-Omar Hospital, Rabwah with provisional diagnosis of neonatal sepsis were recorded. Cases of culture proven sepsis, or probable sepsis were included in this study and were treated with appropriate antibiotics.

Results 469 neonates were included in this study. 136(29\%) cases were having culture proven sepsis and $333(71 \%)$ were cases of probable sepsis. 315(67.2\%) were early onset and 154(22.8\%) were late onset sepsis. 363(77.4\%) cases were discharged, 38(8.1\%) left against medical advice, and $68(14.5 \%)$ died. Mean WBC count of all the cases in this study was 19.756 , median was 17.20 , and standard deviation was 10.883. Mean WBC count in early onset and late onset cases was 20.92 and 17.36 respectively $(p=0.006)$. Mean WBC count in probable sepsis and culture proven sepsis was 19.99 and 19.16 respectively $(p=0.435) .333$ had normal WBC count (5000$30000)$ and in these $41(12.4 \%)$ died. Among 81 cases with WBC count $>30000,18(23 \%)$ died. And in 17 patients with WBC count $<5000,9(53 \%)$ died. $(\mathrm{p}<0.001)$.

Conclusions Majority of cases of neonatal sepsis have normal WBC count. Those with leucopenia suffer highest mortality, followed by those with high WBC count as compared with those with normal WBC count. Cases of early onset have higher WBC count.

\section{3 HOW TO USE C-REACTIVE PROTEIN IN SEPTIC SCREENING OF TERM AND NEAR TERM NEWBORNS?}

doi:10.1136/archdischild-2012-302724.1193

${ }^{1} \mathrm{D}$ Rebelo, ${ }^{1} \mathrm{~T}$ Santos, ${ }^{1} \mathrm{M}$ Albuquerque, ${ }^{1} \mathrm{G}$ Oliveira, ${ }^{2} \mathrm{~T}$ Rodrigues. ${ }^{1} \mathrm{Hospital}$ de Santa Maria; ${ }^{2}$ Biomathematics Department, Lisbon University School of Medicine, Lisbon, Portugal

Background and Aims Early onset sepsis is a serious condition, with challenging diagnosis.

C-Reactive Protein cut-off values for treatment vary according to different authors and protocols from $5 \mathrm{mg} / \mathrm{L}$ to $50 \mathrm{mg} / \mathrm{L}$.

The objective of this study was to determine the CRP cut-off value in septic screening of term and near term NB.

Methods All NB with gestational age $(\mathrm{GA})^{3} 35$ weeks admitted to the nursery of a tertiary hospital in the course of one year, with risk for early neonatal sepsis where included.

We collected data from all analysis (CRP/CBC) until treatment decision, peripheral blood-culture and clinical findings.

A positive septic screening (indicating treatment) resulted from a score involving CRP and leukocy te/neutrophil count.

Newborns were thereafter included in the category "presumption of infection" (POI) if they met at least one of the following criteria: CRP > $50 \mathrm{mg} / \mathrm{L}$; maternal sepsis; NB with positive bloodculture; several positive markers and subtle clinical features; multiple risk factors and subtle clinical features.

Results From 2478 NB admitted, 193 were included, mean GA 38.7 weeks. CRP for untreated NB varied between 10 and $16 \mathrm{mg} / \mathrm{L}$. Those that underwent antibiotic therapy had CRP values between 10 and $151 \mathrm{mg} / \mathrm{L}$

CRP for NB with POI varied between 22 and $151 \mathrm{mg} / \mathrm{L}$, treated but with no late presumption of infection between 10 and $48 \mathrm{mg} / \mathrm{L}$.

A cut-off level of $20 \mathrm{mg} / 1$ would have selected 16 without POI and missed none. 